\title{
Deriving functional astrocytes from mouse embryonic stem cells with a fast and efficient protocol
}

Conference or Workshop Item

Accepted Version

Juneja, D. S., Nasuto, S. and Delivopoulos, E. (2019) Deriving functional astrocytes from mouse embryonic stem cells with a fast and efficient protocol. In: 41st International Engineering in Medicine and Biology Conference, 23-27 July 2019, Berlin, Germany, pp. 2994-2996. doi:

https://doi.org/10.1109/EMBC.2019.8857058 Available at http://centaur.reading.ac.uk/83972/

It is advisable to refer to the publisher's version if you intend to cite from the work. See Guidance on citing.

To link to this article DOI: http://dx.doi.org/10.1109/EMBC.2019.8857058

All outputs in CentAUR are protected by Intellectual Property Rights law, including copyright law. Copyright and IPR is retained by the creators or other 
copyright holders. Terms and conditions for use of this material are defined in the End User Agreement.

\section{www.reading.ac.uk/centaur}

\section{CentAUR}

Central Archive at the University of Reading

Reading's research outputs online 


\title{
Deriving Functional Astrocytes from Mouse Embryonic Stem Cells with a Fast and Efficient Protocol
}

\author{
Deppo S. Juneja, Slawomir Nasuto and Evangelos Delivopoulos* Senior Member IEEE
}

\begin{abstract}
A growing number of studies highlight the structural and functional diversity of astrocytes throughout the central nervous system. These cells are now seen as heterogeneous as neurons and are implicated in a number of neurological and psychiatric diseases. Efficient generation of diverse subtypes of astrocytes can be a useful tool in investigating synaptogenesis and patterns of activity in developing neural networks. In this study, we developed a protocol for the fast and efficient differentiation of astrocytes from mouse embryonic stem cells, as evidenced by the upregulation of genes related to astrocytic development (Gfap, Aldh111). Generated astrocytes exhibit phenotypic diversity, which is demonstrated by the variant expression of markers such as GFAP, ALDH1L1, AQP4 and S100ק, amongst subgroups within the same cell population. In addition, astrocytes exhibited differential calcium transients upon stimulation with ATP. Our protocol will facilitate investigations, regarding the involvement of astrocytes in the structural and functional connectivity of neural networks.
\end{abstract}

\section{INTRODUCTION}

A variety of stem cell based protocols have been suggested, in order to elucidate astrocytogenesis and to provide a source of astrocytes for neural engineering [1], [2] and transplantation studies. The proposed methods derive astrocytes from human induced pluripotent stem cells (hiPSC), via formation of embryoid bodies (EB) and subsequent generation of either neural progenitor cells [3] (NPC) or glial progenitor cells [4] (GPC). Researchers have also generated murine astrocytes from fibroblasts, via small molecule reprogramming [5], and also from neural stem cells (NSC), with the use of BMP4 [6]. Kuegler et al. used a twostep differentiation approach based on heparin supplementation, to first neurally induct mESC and then derive functional murine astrocytes [7]. The protocol produces astrocytes that are inflammatory competent and provide neurotropic support, when co-cultured with neurons. However, the majority of these protocols require considerable time to generate functional astrocytes that express relevant markers (usually more than 60 days). Furthermore, these methods often employ either serum or expensive growth factors to recapitulate the developmental niche. Most importantly, numerous protocols rely solely on the expression of GFAP to mark the completion of astrocyte differentiation

\footnotetext{
* Evangelos Delivopoulos (corresponding author phone: +44 118378 8615; e-mail: e.delivopoulos@ reading.ac.uk) is with the School of Biological Sciences, University of Reading, RG66AY, UK.
}

and maturation. This is not ideal, as specific subtypes of astrocytes are GFAP negative, whereas the subventricular zone (SVZ) contains subpopulations of neural stem cells that are GFAP positive [8]. Therefore, verification of astrocyte differentiation and maturation requires characterization of multiple genes and protein markers, related to astrocytic development. In this study, we have developed a protocol that can produce astrocytes in less than two weeks. These cells express multiple markers related to astrocyte development and demonstrate $\mathrm{Ca}^{2+}$ transients upon ATP stimulation.

\section{MATERIALS \& METHODS}

\section{A. Cell culture}

The mouse embryonic stem (mES) cell line CGR8 was obtained from Sigma (Sigma \& Aldrich, UK). Precharacterization of the CGR8 cell line was performed in our lab, which included the verification of stem cell marker expression and neuronal differentiation. The cells were kept in an undifferentiated state in LIF (Leukemia Inhibitory Factor) supplemented DMEM (Dulbecco's Modified Eagle Medium) media $(10 \%$ Foetal Calf Serum, $1 \%$ Penicillin/Streptomycin, $1 \%$ L-Glutamine, $100 \mu \mathrm{M}$ Mercaptoethanol). The mES cells were passaged and split (ratio 1:8) every 2 days. For differentiation, we adapted a mass suspension protocol by Peljto et al [9]. On day $0 \mathrm{mES}$ cells were seeded on non-tissue culture treated petri-dishes at a density of 50,000 cells $/ \mathrm{mL}$ and allowed to aggregate into embryoid bodies (EB) in ADFNK media (ADMEM/F12:Neurobasal medium (1:1), 10\% Knockout Serum Replacement, 1\% Penicillin/Streptomycin, 1\% LGlutamine, $100 \mu \mathrm{M}$ 2-Mercaptoethanol) without LIF. Media was exchanged with fresh at day 2 and day 5 of differentiation. On day 2, $1 \mu \mathrm{M}$ RA was supplemented into the media. On day 6, EBs were seeded directly onto laminin coated 24 well plates containing astrocyte differentiation media (ADMEM/F12, 2\% FBS, N2, 1\% L-glutamine, 1\% Pen/Strep, $100 \mu \mathrm{M} \beta$-mercaptoethanol and $50 \mu \mathrm{g} / \mathrm{mL}$ heparin [10] (Sigma Aldrich, UK). The cells were assessed via bright field microscopy every other day and cultured for up to 28 days. The media was changed every two days. The entire process is summarized in Figure 1.

\section{B. Immunohistochemistry}

After 1-28 days in vitro (DIV) cell cultures were washed with PBS, fixed with $3.7 \%$ formaldehyde in PBS for 30 minutes 
and permeabilised with $0.02 \%$ Triton $\mathrm{X}-100$ diluted in $10 \%$ goat serum for 15 minutes. Subsequently, cells were washed with PBS and $10 \%$ goat serum was added to block against non-specific binding for 2 hours. Cells were then stained with appropriate primary (Gfap, Aqp4, Aldh111, S100ß, P2X, P2Y) and secondary antibodies. Nuclei were counterstained with H-33342 (Hoechst dye). Primary and secondary antibodies were left on the fixed cultures for 2 hours at room temperature, or overnight at $4^{\circ} \mathrm{C}$. Substrates with stained cultures were mounted in Vectashield ${ }^{\circledR}$. Images were taken on an epifluorescent microscope with x10 and x20 lenses.

\section{C. $R T-P C R$}

Total RNA from 3 separate culture experiments was isolated using the QIAGEN RNeasy Kit according to the manufacturer's instructions, measured in a spectrophotometer and purity ensured by 260/280 $\mathrm{nm}$ ratio of greater than 1.95 for all samples. Each RNA sample was treated with DNase I from the RNeasy Kit, to eliminate any genomic contamination. The QIAGEN QuantiTect Reverse Transcription Kit was used to transcribe $1 \mu \mathrm{g}$ of RNA to cDNA. PCR was conducted with the DreamTaq Green PCR Master Mix (ThermoFisher). The protocol consisted of an initial denaturation step at $95^{\circ} \mathrm{C}$ for 2 min and 35 cycles of: i) denaturation step $30 \mathrm{~s}$ at $95^{\circ} \mathrm{C}$, ii) annealing step $30 \mathrm{~s}$ at $55^{\circ} \mathrm{C}$ and iii) extension step $1 \mathrm{~min}$ at $72^{\circ} \mathrm{C}$. A final extension step of $5 \mathrm{~min}$ at $72^{\circ} \mathrm{C}$ was conducted at the end. Separation of PCR products was performed in a $1 \%$ agarose gel via electrophoresis and visualization was done with SYBR safe DNA gel stain (ThermoFisher). Digital photographs of the gels were taken with the Syngene U Genius 3 System (SLS) and cDNA levels relative to the housekeeping gene Gapdh were calculated using PCR band densitometry (ImageJ NIH National Institutes of Health). Primers for Aqp4 were 5'GCTCAGAAAACCCCTTACCTGTGG-3' (forward) and 5'TTCCATGAACCGTGGTGACTCC-3' (reverse). Primers for Aldh111 were 5'-CTCGGTTTGCTGATGGGGACG-3' (forward) and 5'-GCTTGAATCCTCCAAAAGGTGCGG-3' (reverse). Primers for Gfap were 5'GCCTCGTCCCGTAGACAAAATGGTG-3' (forward) and 5'-GTAGTTGAGGTCAATGAAGGGGTCGTTG-3'

(reverse). Primers for S100 $\beta$ were 5'GGTTGCCCTCATTGATGTCTTCCAC-3' (forward) and 5'CTTCCTGCTCCTTGATTTCCTCCAG-3' (reverse).

\section{Calcium spectrofluorometry}

Astrocyte $\left[\mathrm{Ca}^{2+}\right]_{\mathrm{i}}$ concentration dynamics were evaluated using $\mathrm{Ca}^{2+}$ sensitive fluorescent dye Fluo-4/AM(Molecular Probes). Cells were stimulated pharmacologically with Adenosine Triphosphate (ATP) (Sigma Aldrich, Poole, UK) at $50 \mathrm{uM}$ concentration. Prior to recording, cells loaded with Fluo-4/AM $\left(2.5 \mu \mathrm{M}\right.$ ) for $30 \mathrm{~min}$ at $37^{\circ} \mathrm{C}, 5 \% \mathrm{CO} 2$ (Molecular Probes) [11]-[13]. Subsequently, cells were thoroughly rinsed with Hank's Balanced Salt Solution (HBSS) to remove extracellular traces of the dye and to complete deesterification. In some experiments $100 \mu \mathrm{M}$ Suramin was applied for 30 minutes before imaging. Excitation and emission wavelengths were $494 \mathrm{~nm}$ and $516 \mathrm{~nm}$ respectively. All fluorescence measurements were made at $37^{\circ} \mathrm{C}$ (Warner
Instruments). Changes in $\left[\mathrm{Ca}^{2+}\right]_{\mathrm{i}}$ were detected with an inverted Nikon Eclipse TE2000-S microscope (Nikon) equipped with a xenon arc lamp (Sutter Instruments).

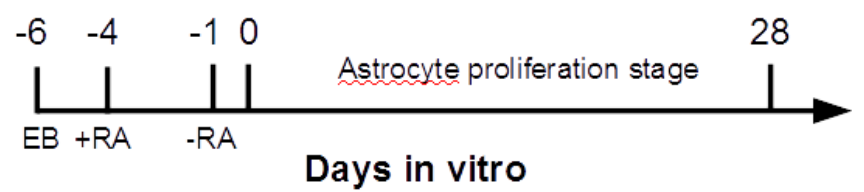

Fig. 1. Timeline of astrocyte differentiation from mouse embryonic stem cells (CGR8) in under 2 weeks.

\section{RESULTS AND DISCUSSION}

\section{A. Genetic analysis of differentiated astrocytes}

We harvested migrating cells on the laminin coated coverslips, extracted total RNA and screened for the genetic expression of astrocytic markers Gfap and Aldh111 via RTPCR. Aldh111 is a pan-astrocytic marker and an early marker of astrocyte maturation [4]. Analysis revealed a significant $(\mathrm{p}<0.05)$ upregulation of Aldh111 from DIV $7(67.5 \% \pm 3.6 \%$, $\mathrm{N}=3)$ to DIV28 $(120.7 \% \pm 27.5 \%, \mathrm{~N}=3$,). Gfap was also upregulated between DIV $7(53 \% \pm 11.9 \%, \mathrm{~N}=3)$ and DIV 28 $(102.9 \% \pm 28.2 \%, \mathrm{~N}=3)$.

We did not detect any expression of neural progenitor gene Nestin later than DIV 7, suggesting absence or very limited presence of neural progenitors, during the EB seeding/astrocyte induction phase of our differentiation protocol (data not shown).
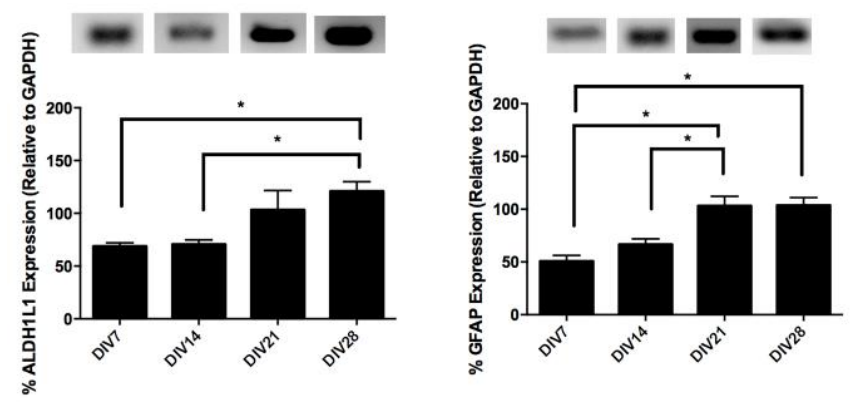

Fig. 2. RT-PCR analysis of total RNA from mESC astrocytes showing expression of Aldh111 and Gfap at DIV 7-28 post astrocyte induction protocol. Data analyzed relative to Gapdh which was the house keeping gene. Representative, individual bands shown are from RNA collected from different cultures. (All data are expressed as mean \pm SEM from three separate experiments, $* \mathrm{P}<0.05)$

\section{B. Expression of protein markers in $m E S C$ derived astrocytes}

We performed a time course study of the expression of established astrocytic proteins, using immunocytochemistry: ALDH1L1, GFAP, S100 $\beta$ and AQP4. Figure 3 depicts examples of positive stains of DIV 28 cultures (Fig. 3 A:ALDH1L1, B:GFAP, C:S100ß, D:AQP4).

Presence and absence of single markers cannot sufficiently describe cell populations. Even though GFAP has been consistently used as a mature astrocytic marker it is not expressed in all astrocytes. For example, there is weak GFAP expression in grey matter protoplasmic astrocytes. Astrocytes can also be identified by the expression of calcium-binding 
protein S100ß, the enzyme ALDH1L1 and the water channel protein AQP4. In our cell populations we observed variant expression patterns of all four markers, verifying the presence of astrocytes at different developmental stages.
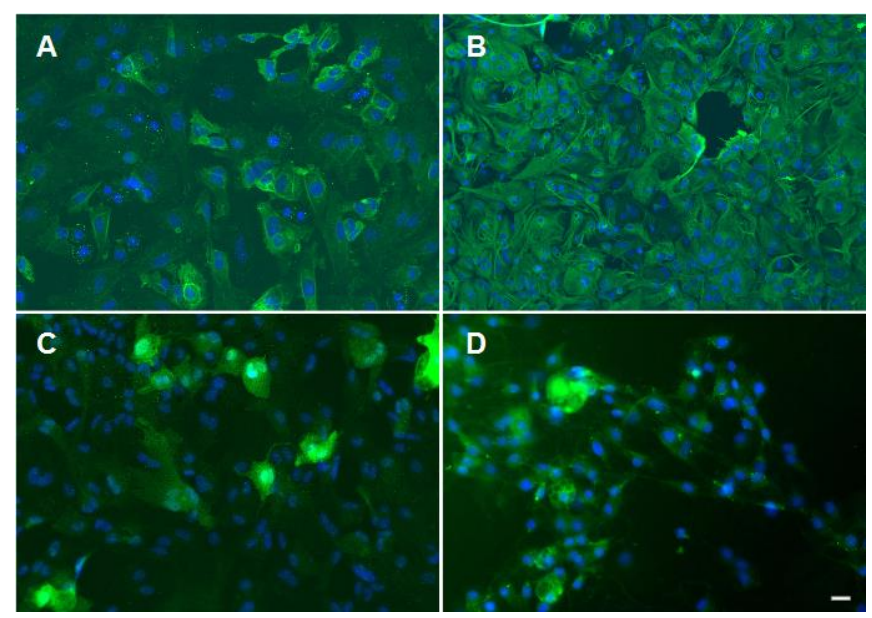

Fig. 3. Epi-fluorescent images of DIV 28 astrocytes inducted from mESC, immunofluorescently labelled for established astrocytic markers: A: ALDH1L1, B: GFAP, C: S100 $\beta$ and D: AQP4. Cell nuclei marker H-33342 (blue stain). Scale bar $20 \mu \mathrm{m}$.

\section{Calcium responses to ATP stimulation in $\mathrm{mESC}$ derived astrocytes}

A time-lapse sequence of a 5-minute recording is illustrated in figure 4, where $\mathrm{Ca}^{2+}$ concentration in multiple regions of interest (e.g. top left and centre (red and yellow arrows), bottom centre (white arrow)) increases and in some cases returns to initial levels. Evoked calcium transients displayed slow kinetics with a rapid rising phase, a peak amplitude and an extended decay phase, as seen in vivo [14]. Responses varied across cultures from different days in vitro, indicating astrocytes at different stages of maturation, or possibly at distinct developmental paths.

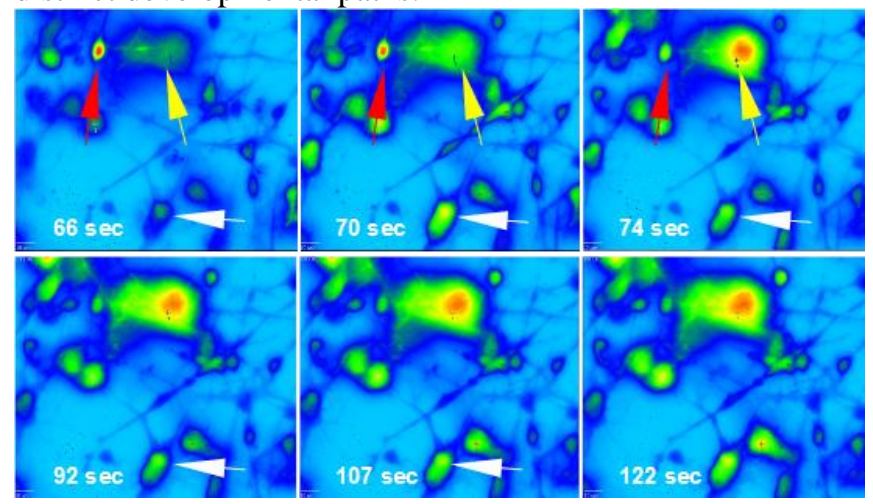

Fig. 4. Response of mESC derived astrocytes, treated with Fluo-4, upon ATP stimulation (60s). The time-lapse heat-map demonstrates cells exhibiting lasting $\mathrm{Ca}^{2+}$ elevation (yellow arrow), biphasic responses (white arrow) and quick response and return to baseline (red arrow). Recording ended at 300s.

\section{CONCLUSION}

In the current study, we differentiated mouse embryonic stem cells (CGR8) into astrocytes. We demonstrate that his can be achieved via a two-step process of EB formation and neuralization, which is followed by an expansion phase in astrocyte inducing media. Our protocol does not require sorting processes [15], or inflammatory stimulants and to our knowledge it is currently the fastest method (13 days) to differentiate astrocytes that express relevant markers and exhibit $\mathrm{Ca}^{2+}$ transients, upon ATP stimulation.

\section{REFERENCES}

[1] C. P. Unsworth et al., "Patterning and detailed study of human hNT astrocytes on parylene-C/silicon dioxide substrates to the single cell level," Biomaterials, vol. 32, no. 27, pp. 6541-6550, 2011.

[2] E. Delivopoulos and A. F. Murray, "Controlled Adhesion and Growth of Long Term Glial and Neuronal Cultures on ParyleneC," PLoS One, vol. 6, no. 9, p. e25411, 2011.

[3] J. Tcw et al., "An Efficient Platform for Astrocyte Differentiation from Human Induced Pluripotent Stem Cells," Stem Cell Reports, vol. 9, no. 2, pp. 600-614, 2017.

[4] R. Santos et al., "Differentiation of Inflammation-Responsive Astrocytes from Glial Progenitors Generated from Human Induced Pluripotent Stem Cells," Stem Cell Reports, vol. 8, no. 6, pp. 1757-1769, 2017.

[5] E. Tian et al., "Small-Molecule-Based Lineage Reprogramming Creates Functional Astrocytes," Cell Rep., vol. 16, no. 3, pp. 781792, 2016.

[6] S. Kleiderman et al., "Functional and phenotypic differences of pure populations of stem cell- derived astrocytes and neuronal precursor cells," Glia, vol. 64, no. 5, pp. 695-715, 2016.

P. B. Kuegler et al., "GFAP-independent inflammatory competence and trophic functions of astrocytes generated from murine embryonic stem cells," Glia, vol. 60, no. 2, pp. 218-228, 2012.

[8] M. Faiz, N. Sachewsky, S. Gascón, K. W. A. Bang, C. M. Morshead, and A. Nagy, "Adult Neural Stem Cells from the Subventricular Zone Give Rise to Reactive Astrocytes in the Cortex after Stroke," Cell Stem Cell, vol. 17, no. 5, pp. 624-634, 2015.

[9] M. Peljto, J. S. Dasen, E. O. Mazzoni, T. M. Jessell, and H. Wichterle, "Functional Diversity of ESC-Derived Motor Neuron Subtypes Revealed through Intraspinal Transplantation," Cell Stem Cell, vol. 7, no. 3, pp. 355-366, 2010.

[10] T. Nagayasu, S. Miyata, N. Hayashi, R. Takano, Y. Kariya, and K. Kamei, "Heparin structures in FGF-2 - dependent morphological transformation of astrocytes," 2005.

[11] J. T. Neary, Y. Kang, Y. Bu, E. Yu, K. Akong, and C. M. Peters, "Mitogenic signaling by ATP/P2Y purinergic receptors in astrocytes: involvement of a calcium-independent protein kinase $\mathrm{C}$, extracellular signal-regulated protein kinase pathway distinct from the phosphatidylinositol-specific phospholipase C/calcium pathway," J. Neurosci., vol. 19, no. 11, pp. 4211-4220, 1999.

[12] K. R. Gee, K. A. Brown, W. N. Chen, J. Bishop-Stewart, D. Gray, and I. Johnson, "Chemical and physiological characterization of fluo-4 Ca(2+)-indicator dyes.," Cell Calcium, vol. 27, no. 2, pp. 97-106, 2000.

[13] G. James and A. M. Butt, "P2X and P2Y purinoreceptors mediate ATP-evoked calcium signalling in optic nerve glia in situ.," Cell Calcium, vol. 30, no. 4, pp. 251-259, 2001.

[14] E. Shigetomi, S. Patel, and B. S. Khakh, "Probing the Complexities of Astrocyte Calcium Signaling," Trends Cell Biol., vol. 26, no. 4, pp. 300-312.

S. H. Yuan et al., "Cell-surface marker signatures for the isolation of neural stem cells, glia and neurons derived from human pluripotent stem cells," PLoS One, vol. 6, no. 3, pp. e17540e17540, Mar. 2011. 